\title{
Comparative Study of OFDM \& MC-CDMA in WiMAX System
}

\author{
Anjali Koranga ${ }^{1}$, Geeta Joshi ${ }^{2}$ \\ ${ }^{1}$ (ECE, BTKIT Dwarahat, Almora, India) \\ ${ }^{2}(E C E$, BTKIT Dwarahat, Almora, India)
}

\begin{abstract}
Recent advances in wireless communication need such kind of techniques which support higher data rate transmission. In this paper study of orthogonal frequency division multiplexing (OFDM) \& multicarrier code division multiple access (MC-CDMA) has been done. MC-CDMA is scheme which works more efficiently in case of mobile environment. A novel approach of Adaptive OFDM is proposed here which provide better SNR performance.
\end{abstract}

Keywords: AMC, MC-CDMA, OFDM, WiMAX, Adaptive OFDM

\section{INTRODUCTION}

Wireless communications have become very pervasive. The number of mobile phones and wireless internet user has been increased in recent years. The objectives are to develop a system for anytime, anywhere, and anyone. These systems are known as beyond 3G. These 3G beyond systems includes "higher data rates," "improved roaming" and "true inter-system mobility management". World Wide Interoperability for Wireless Access (WiMAX) comes under the system beyond 3G. It is a new wireless OFDM-based technology that provides high quality broadband service. It includes the definition of both MAC layer \& Physical layer. The main performance becomes more challenging when mobile environments are encountered in wireless channel. Physical layer is flexible in such a way that researchers can modulate it according to the system requirement. One way is adaption of OFDM. OFDM is a popular DFT-based technique initially proposed in the 1970s [1]. Its main use was providing bandwidth reduction. We can say it as an alternative to conventional multicarrier techniques such as FDM. OFDM has gained popularity with the emergence of wireless communications and wideband systems because of its inherent ability to compensate for multipath. In1993 OFDM is combined with code-division multiplexing (CDM) and proposed a new modulation scheme, MC-CDMA. MC-CDMA effectively mitigates multipath interference while providing multiple access capability.

Next sections of this article are arranged as follows. The next section provides description about OFDM and MC-CDMA system, a brief overview of AMC scheme. Adaptive modulated OFDM are then presented, followed by concluding remarks.

\section{ORTHOGONAL FREQUENCY DIVISION MULTIPLEXING (OFDM)}

OFDM is a digital modulation scheme in which a wideband signal is split into a number of narrowband signals. It is a multicarrier transmission technique, which divides the bandwidth into several parallel subcarriers. Each one sub-carrier is modulated by a low rate data stream. OFDM uses the principles of FDM to allow multiple messages to be sent over a single radio channel. We can say it provides an improved spectral efficiency. In conventional FDM, adjacent channels are well separated using a guard interval, which is just the wastage of bandwidth. In order to realize the overlapping technique, crosstalk between adjacent channels must be reduced. Therefore, orthogonality between subcarriers is required.

Random data generator is used to generate a serial random binary data. This binary data stream represents the raw information that going to be transmitted. The serial binary data is then fed into OFDM transmitter. The modulated serial symbols are converted to parallel symbols using serial to parallel convertor $(\mathrm{S} / \mathrm{P})$.The parallel symbols are then transmitted in parallel by assigning each symbol to one subcarrier in the transmission. An IFFT is used to find the corresponding time waveform. The guard period is then added to the start of each symbol. The length of guard period is chosen based on the WiMAX technology standard, which is 25 percent of the length of symbol. 


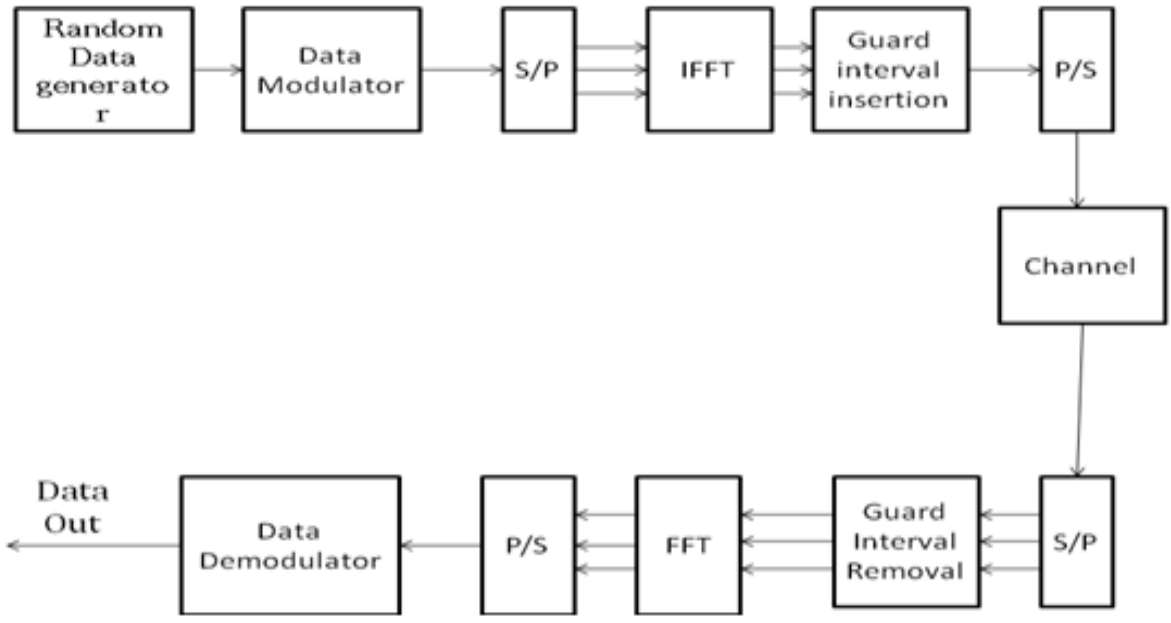

Fig 1: OFDM based system

Guard time is introduced to eliminate intersymbol interference (ISI) caused by delay spread. This can take care of ISI, but intercarrier interference (ICI) (crosstalk between subcarriers) remains an issue. To reduce ICI, OFDM symbols are cyclically extended into the guard interval. This cyclic extension ensures that an OFDM symbol will have an integer number of cycles in the DFT interval as long as the delay is less than the guard time. To overcome the unknown phase and amplitude ambiguities, two techniques, coherent and differential detection, are used [2].

\section{MULTiCARRIER CDMA}

MC-CDMA is a hybrid approach in which OFDM \& CDMA are implemented together. MC-CDMA signal is made up of a series of equal amplitude subcarriers. However, each sub-carrier transmits a different symbol unlike OFDM. The MC-CDMA transmitter can be implemented by combining a spreader and despreader before and after OFDM Rx \& Tx respectively. The input data sequence is first converted into a number of parallel data sequences. Then each stream is modulated with by a suitable digital method, depending upon the current channel signal to noise ratio estimate information provided by the receiver. After this the modulated bit streams are passed through the Multi-carrier CDMA system transmitter then this is up-converted by an RF amplifier and finally transmitted through the channel. At the receiver site reverse operations are performed to demodulate and decode the original information. In MC-CDMA orthogonal walsh-Hadamard code are used for spreading of the signal [3].

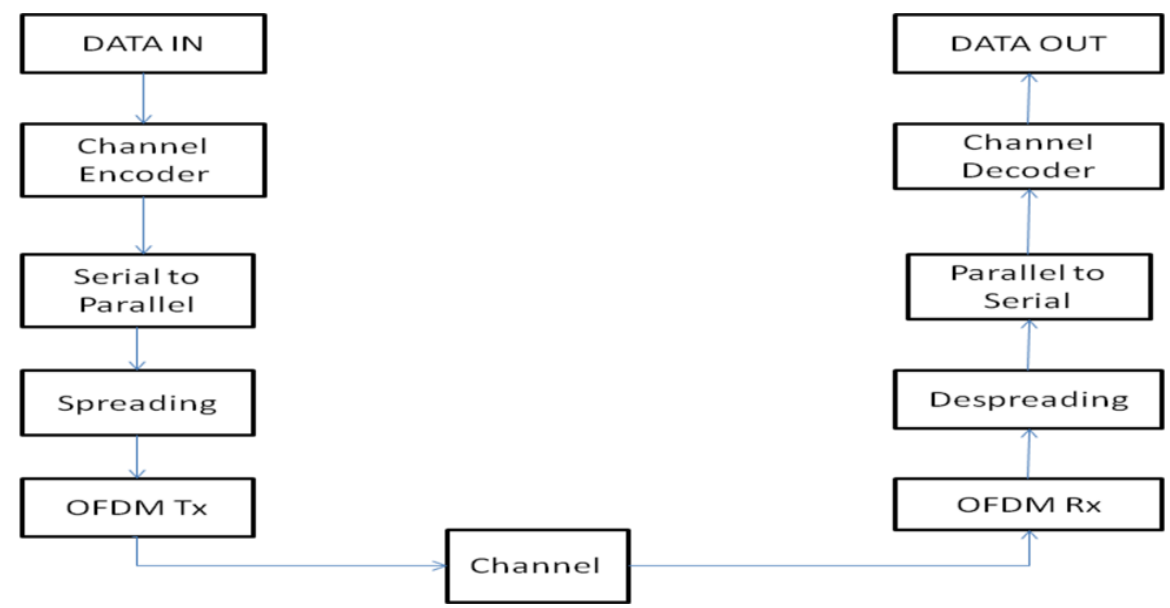

Fig 2: MC-CDMA based system

\section{ADAPTIVE MOdULATION SCHEME}

The use of Adaptive Modulation and Coding (AMC) is one of the techniques for WiMAX system that have been developed to achieve high spectral efficiency on fading channels. The main idea of AMC is to dynamically change the Modulation and Coding Scheme (MCS) according to the channel condition. The decision about selecting the appropriate MCS is performed at the receiver side according to the observed channel condition with the information fed back to the transmitter in each frame. Using adaptive modulation scheme, WiMAX system can switch to the highest order modulation depending on the channel conditions. As 
the signal-to-noise ratio (SNR) is very good near the BS, so higher order modulation scheme is used in this area to increase the throughput. While for the areas where the SNR is poor the system switches to the lower order modulation scheme to maintain the connection quality and link stability with increased throughput. The supported modulations are BPSK, QPSK, 16- QAM and 64-QAM. By using this scheme we can enhance the system performance.

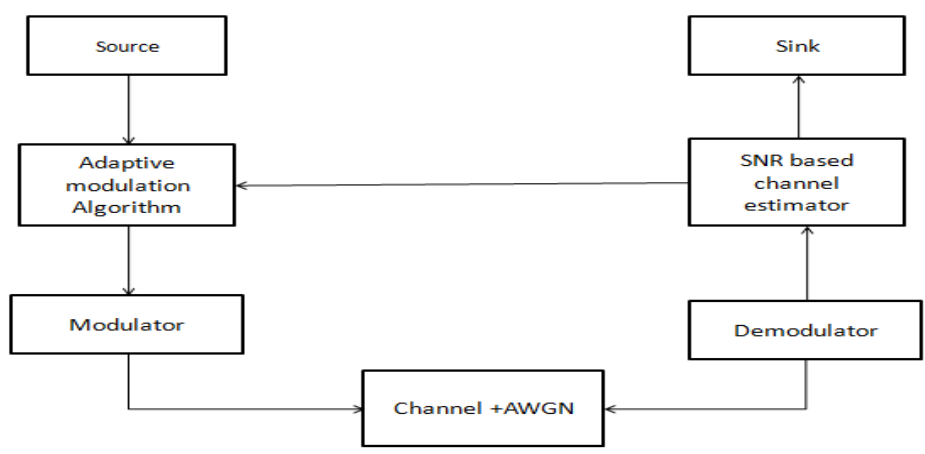

Fig 3: Adaptive modulation System

In WiMAX adaptive modulation based OFDM has used for better system throughput as well as spectral efficiency. However, when this modulation is used in conjunction with MC-CDMA it gives very assuring way to increase data rate. In current scenario demand of a high data rate can be achieved by this scheme even in the mobile environment.

\section{ADAPTIVE MOdULATED OFDM SYSTEM}

Below is the figure for Adaptive Modulated OFDM system. It consists of Adaptive modulation switch. The input serial binary data stream is formatted into word size depend on the modulation type required for transmission, for example 4 bits/symbol for 16-QAM. At the front end of the receiver the SNR is determined. This information is directly fed to the mode selector as shown in Figure 4.Based on the information which is the instantaneous SNR the mode selector selects the modulation scheme which satisfies the switching threshold. Again the mode selector informs the transmitter and receiver to use the selected modulation scheme. At the transmitter and receiver the adaptive modulator and demodulator blocks consists of different modulators and demodulators which are used to provide different modulation and demodulation orders respectively.

Similarly adaptive modulation based MC-CDMA system can be used in the physical layer of WiMAX system. MC-CDMA provides better BER vs. SNR as compared to OFDM based system.

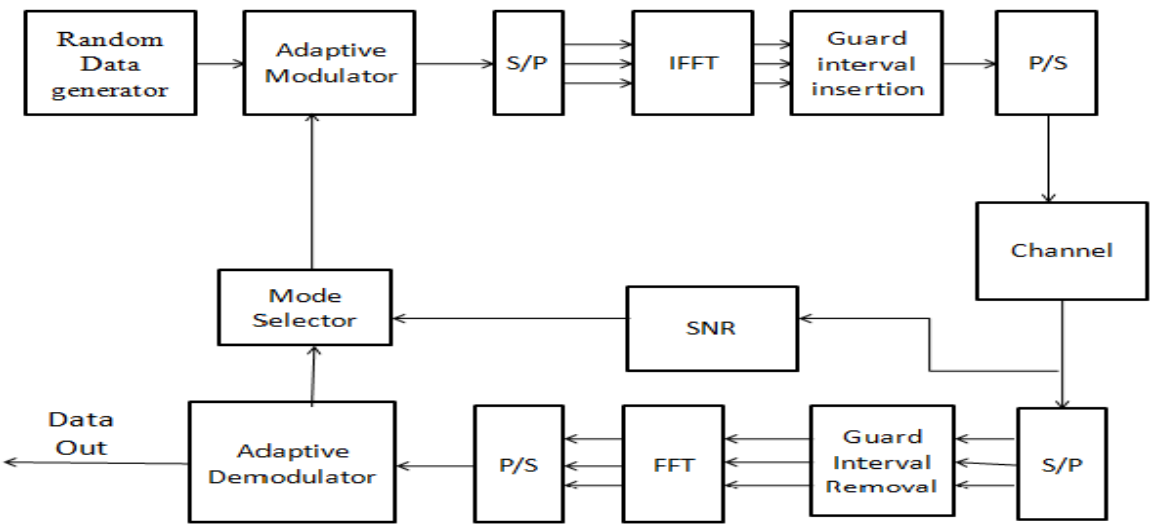

Fig 4: Adaptive Modulated OFDM based system

\section{SIMULATION RESUlTS \& DiscuSSION}

The number of bit errors is the number of received bits of a data stream over a communication channel that has been altered due to noise, interference, distortion or bit synchronization errors. The bit error probability pe is the expectation value of the BER. If the medium between the transmitter and receiver is good and the signal to noise ratio is high, then the bit error rate will be very small possibly insignificant and having no noticeable effect on the overall system However if noise can be detected, then there is chance that the bit error rate will need to be considered. The main reasons for the degradation of a data channel and the corresponding bit error rate, BER is noise and changes to the propagation path. 


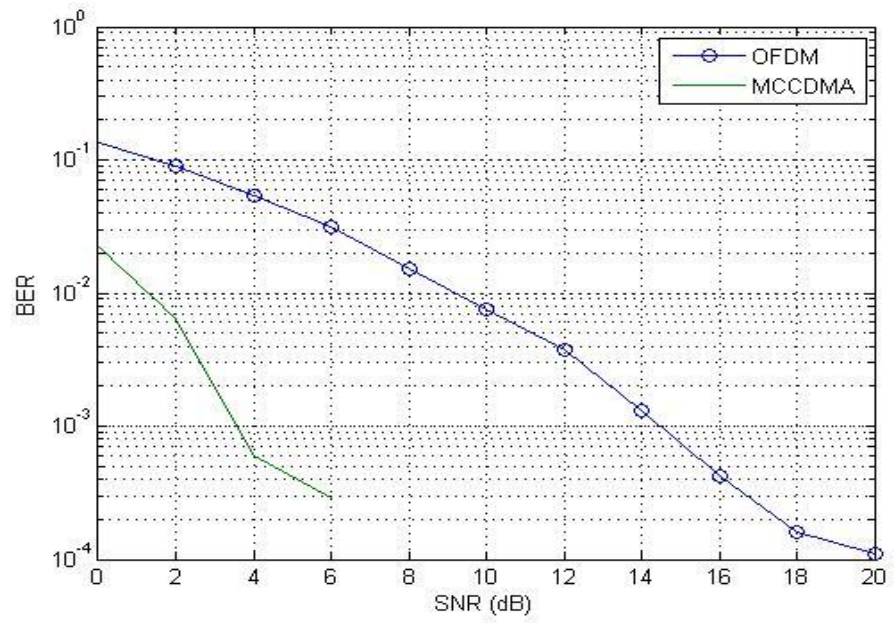

Fig 5: BER vs SNR performance of OFDM \& MC-CDMA scheme for BPSK

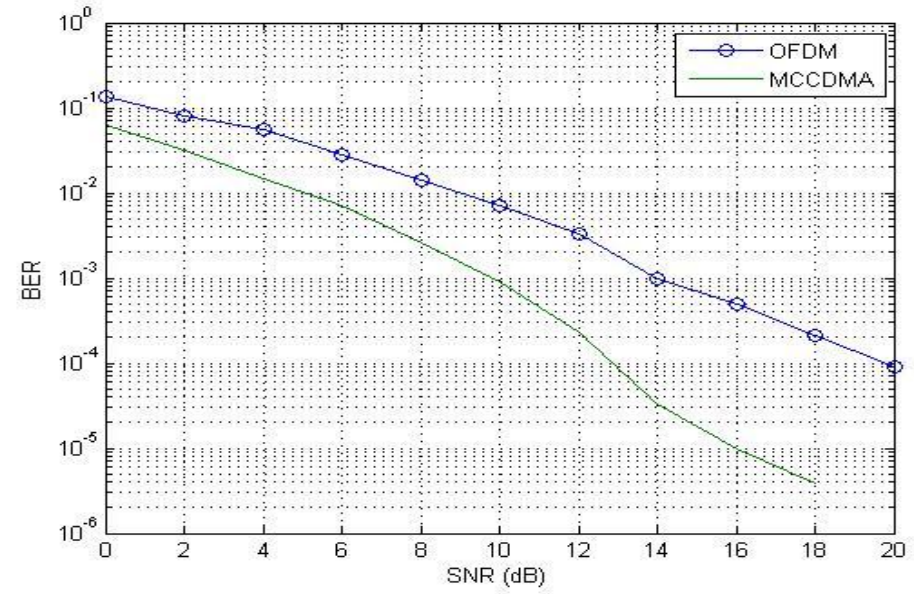

Fig 6: BER vs SNR performance of OFDM \& MC CDMA scheme for QPSK

MC-CDMA is a scheme useful when mobility is added with the system. As with mobile environment signal fading occurs that results in degradation of the performance of the system. This combined scheme of OFDM \& CDMA (MC-CDMA) provides better BER performance.

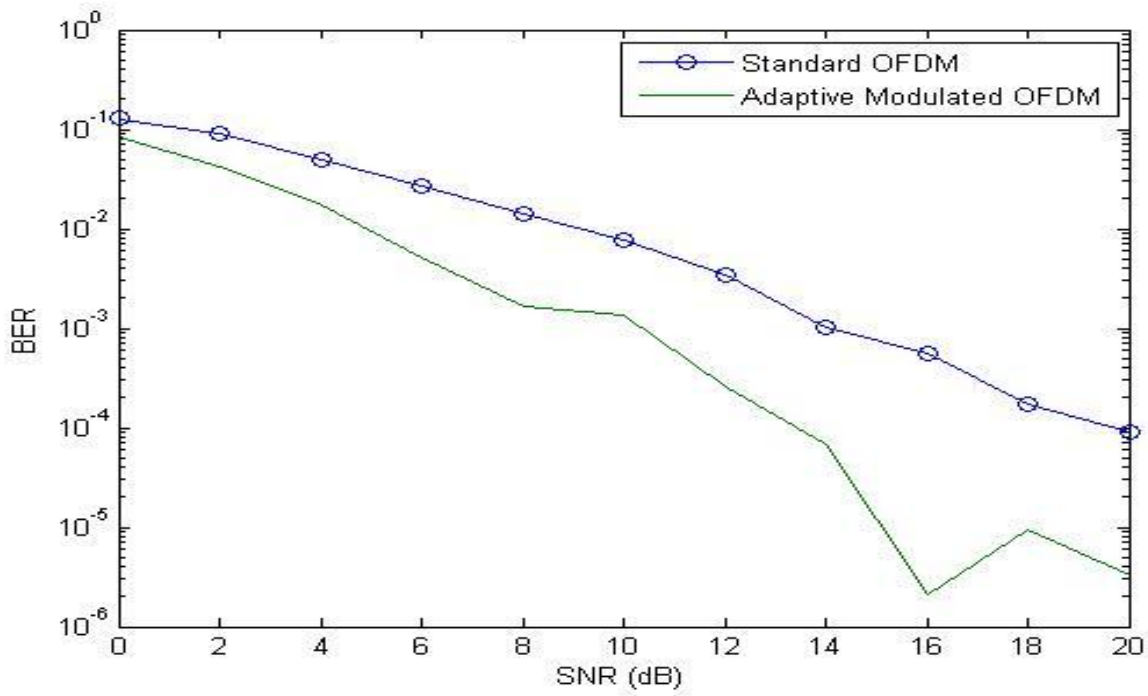

Fig 7: BER vs. SNR performance of OFDM \& Adaptive modulated OFDM scheme 
In the physical layer of WiMAX adaptive modulated OFDM system is included in place of simple OFDM system. In adaptive modulation technique modulation scheme and convolution code rate are varied according to the variation in the communication channel. The transmitter can choice the appropriate modulation scheme and code rate is depend upon the SNR (signal to noise ratio) threshold. Figure.6. Shows the performance of different modulation schemes (BPSK, QPSK, 16QAM, 64QAM) with code rate 1/2, 2/3 \& 3/4 in the AWGN channel evaluated by plotting the BER Vs SNR characteristics.

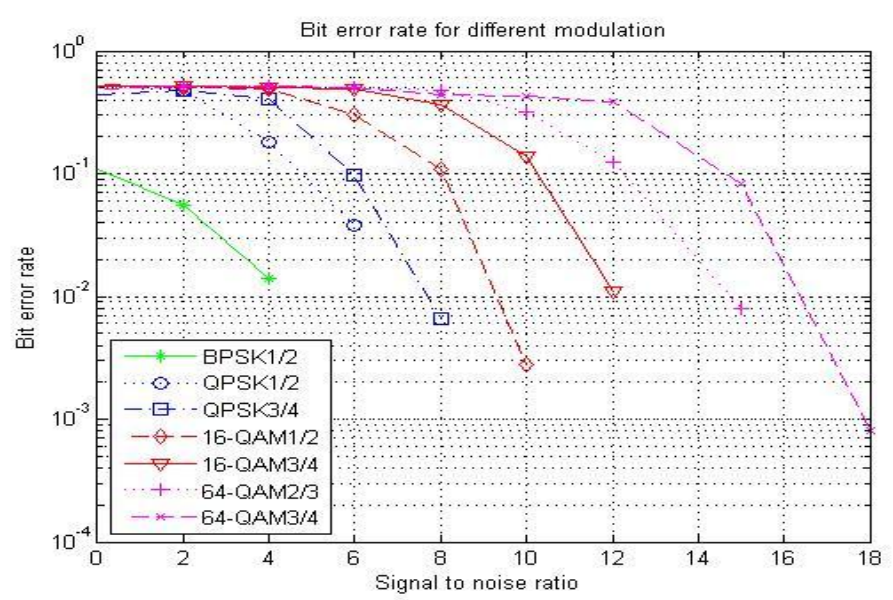

Fig.8. BER Vs SNR for different modulation scheme

\section{Conclusion}

BER performance of OFDM \& MC-CDMA is investigated here. MCCDMA is best suited for the mobile environment. It gives the better BER performance in such conditions. Along with that adaptive modulated OFDM is also compared with the standard OFDM which gives better BER performance than the simple OFDM. Adaptive OFDM is the scheme in which transmitter has freedom to adapt any modulation scheme based on the SNR condition. Further with this if we use adaptive modulation based MC-CDMA system in WIMAX it may provide the much better performance.

\section{REFERENCES}

[1] J.Faezah, and K.Sabira, “ Adaptive Modulation for OFDM Systems”, International Journal of Communication Networks and Information Security (IJCNIS) Vol. 1, No. 2, August 2009.

[2] L. Hanzo et al., "OFDM and MC-CDMA for Broadband Multi-User Communications", Wiley, 2003.

[3] Vinit Grewal and Ajay K. Sharma "Performance Evaluation of WiMAX Network with AMC and MCCDMA for Mobile Environments", International Journal of Multimedia and Ubiquitous Engineering Vol. 7, No. 4, October, 2012 